\title{
Diacronie
}

Studi di Storia Contemporanea

$\mathrm{N}^{\circ} 21,1$ | 2015

Le città di Babele

\section{Anna Tonelli, Gli irregolari. Amori comunisti al tempo della Guerra fredda}

\section{Gianluca Scroccu}

\section{Q OpenEdition \\ Journals}

Edizione digitale

URL: http://journals.openedition.org/diacronie/1934

DOI: $10.4000 /$ diacronie.1934

ISSN: 2038-0925

Editore

Association culturelle Diacronie

Notizia bibliografica digitale

Gianluca Scroccu, «Anna Tonelli, Gli irregolari. Amori comunisti al tempo della Guerra fredda », Diacronie [Online], $\mathrm{N}^{\circ}$ 21, 1 | 2015, documento 12, Messo online il 01 mars 2015, consultato il 23 septembre 2020. URL : http://journals.openedition.org/diacronie/1934 ; DOI : https://doi.org/10.4000/diacronie. 1934 


\section{Diacronie}

\section{RECENSIONE:}

\section{Anna TONELLI, Gli irregolari. Amori comunisti al tempo della Guerra fredda, Roma-Bari, Laterza, 2014, 192 pp.}

a cura di Gianluca SCROCCU *

Anna Tonelli - docente di storia contemporanea, storia dei partiti e dei movimenti politici e storia del giornalismo presso l'Università degli studi di Urbino «Carlo Bo» - è una studiosa attenta e una profonda conoscitrice della storia politica italiana, analizzata non solo attraverso il classico approccio istituzionale, ma anche con uno sguardo critico capace di utilizzare gli strumenti di analisi della storia culturale, con un'attenzione particolare verso l'uso politico dei sentimenti e delle emozioni inteso come modo per costruire identità ${ }^{1}$.

Il saggio Gli irregolari - articolato in cinque capitoli - si inserisce in questo percorso, arricchendolo grazie all'importante contributo apportato alla storiografia sul PCI e sulla sua socialità. Per raggiungere questo obiettivo l'autrice ha condotto un'importante ed originale ricerca archivistica non solo sui documenti del PCI conservati presso la Fondazione Istituto Gramsci, dove ha esaminato l'ancora inedito fondo D'Onofrio, ma anche l'Archivio storico del Ministero degli Esteri e l'archivio del Tribunale commissariale civile e penale della Repubblica di San Marino. Documenti archivistici che nel libro sono fatti sempre interagire con un numero significativo di fonti a stampa della pubblicistica comunista e anche delle forze avverse al partito di Togliatti, oltre che con la principale letteratura storiografica sul tema.

Anna Tonelli dedica il primo capitolo ad una ricostruzione delle unioni sentimentali e degli amori comunisti nati durante gli anni della clandestinità antifascista, un

\footnotetext{
${ }^{1}$ Tra i suoi lavori più recenti sul tema si possono citare TONELLI, Anna, Politica e amore. Storia dell'educazione ai sentimenti nell'Italia contemporanea, Bologna, Il Mulino, 2003; ID., Comizi d'amore. Politica e sentimenti dal '68 ai Papa boys, Roma, Carocci, 2007; ID., Stato spettacolo. Pubblico e privato dagli anni '8o a oggi, Milano, Bruno Mondadori, 2010; ID., Falce e tortello. Storia politica e sociale delle Feste dell'Unità (1945-2011), Roma-Bari, Laterza, 2012.
} 
momento importante in quanto la sfera affettiva del rapporto matrimoniale venne sostituita da quella relativa all'impegno per la realizzazione della causa suprema ovvero l'opposizione alla dittatura mussoliniana ${ }^{2}$. I legami sentimentali di chi era impegnato nella lotta per il rovesciamento del regime furono così vincolati alla volontà di proseguire senza tentennamenti verso la liberazione del paese e l'ottenimento del massimo risultato per il proprio partito.

I problemi si posero nel momento in cui, terminata la guerra, si entrò nel contesto democratico della neonata Repubblica, dove la sfera affettiva poté riacquistare una sua specificità al di là della battaglia ideologica. È in quest'ambito, come si esplicita nel secondo capitolo, che si devono inquadrare le unioni irregolari dei dirigenti e militanti comunisti: un aspetto destinato ad ingenerare problemi molto seri all'interno del partito e verso l'esterno per gli attacchi degli avversari politici, ad iniziare dalla Democrazia Cristiana e dalla stampa avversa al PCI. Il vissuto e la moralità dei comunisti3, indagati in un'ottica che nel volume si allarga per cerchi concentrici dal centro alla periferia, diventarono così, da una parte componente integrante della polemica e della lotta politica generale, dall'altra discrimine all'interno delle relazioni tra dirigenti e iscritti da intendersi come modalità di vivere la propria esistenza nel nuovo stato repubblicano tra condizionamenti morali del passato e volontà di aprirsi alla modernità dei nuovi rapporti sociali.

Ciò permette alla Tonelli di dimostrare come gli approcci sul tema dell'irregolarità dei sentimenti fossero influenzati tanto dalla morale comune quanto da quelli che erano i condizionamenti dell'esistenza quotidiana, ad esempio i retaggi familiari o del contesto locale. Il PCI, nonostante la sua pretesa alterità anche sul piano morale, non era evidentemente un corpo astratto dal tessuto sociale italiano anche se si presentava come alternativo nella sua diversità ideologica e nell'approccio ideologico internazionalista. I suoi militanti finivano infatti per vivere le loro esistenze confrontandosi con luoghi comuni, moralismi e pregiudizi ampiamente diffusi anche nel tanto vituperato contesto borghese.

In questo senso nel libro richiama opportunamente il confronto con la cultura cattolica: le due subculture politiche, quella comunista e quella democristiana, da questo punto di vista si muovevano negli anni della Guerra Fredda su un piano di convergenza in relazione all'osservanza dei principi della morale e della tenuta delle

\footnotetext{
2 TONELLI, Anna, Gli irregolari. Amori comunisti al tempo della Guerra fredda, Roma-Bari, Laterza, 2014, p. 35.

3 BELLASSAI, Sandro, La morale comunista. Pubblico e privato nella rappresentanza del PCI (1947-1956), Roma, Carocci, 2000; CASALINI, Maria, Famiglie comuniste. Ideologie e vita quotidiana nell'Italia degli anni Cinquanta, Bologna, Il Mulino, 2010.
} 
pulsioni e degli istinti sentimentali. Tematiche come quella delle relazioni extraconiugali, della convivenza fuori dal legame ufficiale del matrimonio o ancora l'eccessiva disinvoltura nel vissuto delle relazioni tra sessi erano giudicate da entrambe le sponde con severità e rigorismo, con punte di asperità di giudizio e cesura che a volte nel PCI superavano gli atteggiamenti dei democristiani. Il serrare le fila sul piano della morale, come spiega Tonelli, corrispondeva alla necessità di bloccare i tentativi della Democrazia Cristiana di presentare i comunisti come i "senza Dio e senza famiglia", ovvero come i fautori di un ordine disgregato e licenzioso dove tutto era possibile e dove potevano saltare i vincoli, ad iniziare da quello familiare, su cui si basava la società. Era un rischio che il PCI non poteva permettersi e su cui si giocava la possibilità per il partito di conquistare nuovi consensi in un elettorato dove era forte l'impronta dell'educazione cattolica.

All'interno di questo contesto la disciplina che doveva regolare la vita del militante si riverberava quindi anche nella vita personale: chi andava oltre, cedendo a tentazioni borghesi, faceva emergere un cattivo esempio che, come tale, doveva essere censurato. Il problema si poneva però quando a venire coinvolti in relazioni sentimentali irregolari o che rompevano legami ufficiali nati nel periodo della lotta antifascista erano i massimi dirigenti del partito di sesso maschile: su questo piano la logica della doppia morale e del maschilismo vincevano sull'ortodossia e il principio di uguaglianza tra gli iscritti.

Come si legge nel terzo capitolo, diversi furono i matrimoni nati negli anni della clandestinità andati in frantumi dopo la Liberazione e sostituiti da nuove unioni spesso tra importanti dirigenti e giovani compagne. Nuovi rapporti che palesavano una discriminazione forte non soltanto fra ciò che era consentito ai leader rispetto ai semplici militanti, ma soprattutto tra uomini e donne. Furono donne come Rita Montagnana o Teresa Noce, note anche per la pubblicità critica che venne portata avanti dalla stampa anticomunista sulle loro vicende, a dover subire l'onta del ridimensionamento e dell'umiliazione dopo che i loro compagni avevano deciso di abbandonarle. Dopo la separazione, infatti, spesso si apriva un momento di ridimensionamento dagli incarichi politici per le moglie ripudiate. Come scrive la Tonelli, «con abilità e cinismo politico, i leader del PCI prendono a pretesto le reazioni delle compagne come posizioni contrarie al codice etico comunista, al fine di relegare a compiti secondari le protagoniste di una stagione passata»4.

La rottura matrimoniale per i dirigenti del PCI, o per intellettuali vicini al partito come Gerratana, Einaudi e Vittorini, passava inesorabilmente tramite l'escamotage del 
divorzio da raggiungere tramite i tribunali di San Marino, data la lontananza di quelli ungheresi e bulgari, e viste le lacune della legislazione italiana che prima del 1970 non prevedeva il divorzio. Un traguardo non facile da ottenere, in quanto doveva passare tramite l'istruzione di pratiche sbrigative e fittizie ad esempio in relazione al dover prendere la residenza nel piccolo stato5.

È all'interno di questo orizzonte freddamente burocratico, dove certo c'era poco spazio per il rispetto e il tentativo di non ferire il coniuge da cui ci si voleva distaccare, che si può comprendere quella parte del volume dove si analizzano le modalità attraverso le quali una personalità importante della storia comunista come Teresa Noce fu pesantemente censurata e redarguita dal suo partito nel momento in cui manifestò sulla stampa "borghese" la sua giusta contrarietà ad un divorzio richiesto a sua insaputa dal marito Luigi Longo, legato da tempo a Bruna Conti, e di cui era venuta a sapere solo da un trafiletto del «Corriere della Sera». A San Marino, però, si rivolse non solo Longo. Merito della Tonelli è quello di aver ritrovato le carte che attestano come anche Togliatti abbia tentato questa via, frenato non solo dalla necessità di prendere la cittadinanza sanmarinese, dopo la quale avrebbe perso la sua carica di parlamentare e la sua eleggibilità in Italia, ma anche dalla contrarietà di una combattiva Rita Montagnana, oppostasi all'umiliazione di sottoscrivere motivazioni che dovevano giustificare l'annullamento del matrimonio relative alla sua persona le quali erano assolutamente false ${ }^{6}$. E nell'iter verso la separazione, avvenuta nel pomeriggio del 9 luglio 1951, il libro dimostra con un approccio innovativo come il tutto venne soppesato in maniera certosina anche sul piano procedurale: la Montagnana operò, infatti, in maniera tale da non farsi dettare i vari passi della divisione coniugale dall'ex compagno.

Significativa è poi l'analisi che la Tonelli compie su un altro importante dirigente del partito come Edoardo D’Onofrio, la cui vicenda è esemplificativa sia per il fatto che fu la moglie ad abbandonarlo per un altro compagno da cui ebbe un figlio mentre il marito era in carcere per la sua opposizione al fascismo, sia perché la sentenza di annullamento di San Marino non venne delibata in Italia, per cui i due coniugi dovettero aspettare l'introduzione della legge sul divorzio per sciogliere definitivamente la loro unione. La storia del percorso di annullamento matrimoniale di D’Onofrio è

\footnotetext{
5 Rispetto alla questione della mancata residenza a San Marino, la Tonelli nota alle pp. 115-116 del suo lavoro come tale pratica non fu messa in atto da un esponente vicino ai socialdemocratici come lo storico Aldo Garosci, che vi avrebbe soggiornato mentre era in atto la pratica di annullamento matrimoniale dalla sua consorte, un periodo proficuo anche sul piano intellettuale e della ricerca visto che in quel lasso di tempo avrebbe approfondito lo studio della storia sanmarinese da cui avrebbe tratto il volume intitolato San Marino. Mito e storiografia tra i libertini e il Carducci.

6 TONELLI, Anna, Gli irregolari, cit., pp. 71-84.
} 
ricostruita da Anna Tonelli attraverso l'esame di una lettera, finora inedita, che egli scrisse al presidente del Tribunale di Roma ricostruendo tutte le fasi della sua vicenda, documento interessante anche perché vi emerge l'auto rappresentazione del militante comunista dalla perfetta integrità, pronto a sacrificare i propri affetti per salvaguardare l'onore del partito7.

La morale comunista richiedeva del resto una severa disciplina, spesso spietata, con la Commissione Centrale di Controllo (la «famigerata Ccc», come la definisce la Tonelli nel quarto capitolo) ${ }^{8}$. Coadiuvata dalla Commissione Quadri e dai Comitati esecutivi locali e basata su un gerarchizzato rapporto verticale dal centro alla base, pronta a colpire chi violava i codici interni, sia relativi al tradimento politico da intendersi come messa in discussione radicale della linea ufficiale del partito, sia in relazione alla propria condotta morale. La vigilanza interna, in questo contesto, portava tutti gli iscritti ad essere dei potenziali agenti di sorveglianza della salvaguardia del buon costume del partito. Come si dimostra nel volume, l'indegnità politica e nei comportamenti poteva essere utilizzata non soltanto contro chi contestava il centralismo democratico o l'impossibilità di dissentire rispetto alla linea ufficiale italiana o di Mosca, ma anche riguardo agli "invertiti”, ovvero gli omosessuali. L'accusa di indegnità morale poteva così diventare l'anticamera per dure reprimende e sanzioni, sino alla vera e propria espulsione dal partito. Una pratica particolarmente forte negli anni Cinquanta, di cui rimase vittima ad esempio Pier Paolo Pasolini nel 1949, criticata però successivamente per la sua logica eccessivamente costrittiva e di chiusura ${ }^{9}$.

Il confine tra pubblico e privato era quindi assai sottile tra le file del partito "nuovo" di Togliatti: una doppiezza non soltanto rispetto alla politica internazionale, ma anche nella sfera dei sentimenti. Diverso era però il grado di tolleranza a seconda che si fosse un dirigente, un quadro o un semplice militante di base: «mentre la dottrina impone un comportamento esemplare, la pratica dimostra sovente una deroga riservata a chi occupa posizioni e ruoli di rilievo» ${ }^{10}$. Chi derogava dalle leggi della moralità comunista doveva essere rimesso sulla retta via, anche tramite una pratica inquisitoria e processuale che però non mancava di esempi dove si usava una mano troppo morbida nei confronti di dirigenti di provincia che eccedevano nelle loro attenzioni verso l'altro sesso anche quando venivano spostati di federazione ${ }^{11}$.

Significativo, in questo viaggio all'interno delle diverse forme della "moralità" irregolare dei comunisti negli anni della Guerra Fredda, è poi il quinto e conclusivo

\footnotetext{
7 Ibidem, pp. 97-107.

8 Ibidem, p. 117.

9 Ibidem, pp. 137-139.

${ }^{10}$ Ibidem, p. 59.

${ }^{11}$ Ibidem, pp. 128-136.
} 
capitolo, dove viene analizzato come sia la stampa avversaria ai comunisti, sia quella legata al PCI come «Vie Nuove», si soffermassero sulle pratiche dei comportamenti di dirigenti e semplici militanti del partito di Togliatti. Si veda in proposito il ruolo della posta dei lettori di periodici come «Vie Nuove» o «Noi donne»: i modelli che venivano presentati alle lettrici erano quelli della militante dedita alla causa del partito e a quella familiare, in un orizzonte morale che non ammetteva deroghe o troppa indipendenza e che subiva inevitabilmente l'influsso del cattolicesimo.

In conclusione non si può, dunque, che ribadire l'interesse e l'importanza di questo lavoro di Anna Tonelli. La sua analisi delle tematiche relative all'utilizzo dei sentimenti e della sfera privata come motivo del contendere della lotta politica e del proprio vissuto di militante rappresentano uno strumento valido per interpretare lo spazio pubblico italiano in un frangente complesso come quello della fase più "calda" della Guerra Fredda. Conoscere quanto abbia inciso tale fattore in quegli anni è pertanto fondamentale anche per comprendere quanto venuto nei decenni successivi. Non a caso, come si è visto nella transizione incompiuta dalla Prima alla cosiddetta Seconda Repubblica, il privato è entrato prepotentemente al centro della competizione fra partiti sempre più liquidi e postmoderni anche nel campo della sinistra. 


\section{* L'autore}

Gianluca Scroccu si è laureato in Lettere nel 2000 presso l'Università degli Studi di Cagliari; nel 2005 ha conseguito il dottorato in Studi storici per l'età Moderna e Contemporanea presso l'Università di Firenze. Attualmente è borsista presso il Dipartimento di Storia, Beni culturali e Territorio dell'Università degli Studi di Cagliari. Ha conseguito l'Abilitazione Scientifica Nazionale a professore associato in storia contemporanea. Si occupa principalmente di storia politica, con particolare attenzione alla storia della sinistra italiana, del pensiero di Piero Gobetti, di storia della globalizzazione, di storia della Sardegna e di storia di genere. Su questi temi è autore di volumi e saggi; la sua più recente monografia è Alla ricerca di un socialismo possibile. Antonio Giolitti dal Pci al Psi (Roma, Carocci, 2012).

URL: < http://www.studistorici.com/progett/autori/\#Scroccu >

\section{Per citare questo articolo:}

SCROCCU, Gianluca, «Recensione: Anna TONELLI, Gli irregolari. Amori comunisti al tempo della Guerra fredda, Roma-Bari, Laterza, 2014, 192 pp.», Diacronie. Studi di Storia Contemporanea : Le città di Babele, 29/3/2015, URL:< http://www.studistorici.com/2015/03/29/scroccu_numero_21/ >

\section{Diacronie Studi di Storia Contemporanea 3 www.diacronie.it}

Risorsa digitale indipendente a carattere storiografico. Uscita trimestrale. redazione.diacronie@hotmail.it

Comitato di redazione: Jacopo Bassi - Luca Bufarale - Elisa Grandi - Deborah Paci - Fausto Pietrancosta - Matteo Tomasoni - Luca Zuccolo

Diritti: gli articoli di Diacronie. Studi di Storia Contemporanea sono pubblicati sotto licenza Creative Commons 2.5 . Possono essere riprodotti a patto di non modificarne i contenuti e di non usarli per fini commerciali. La citazione di estratti è comunque sempre autorizzata, nei limiti previsti dalla legge. 